\title{
The Biochemistry Show: a new and fun tool for learning
}

Ono, A.H. ${ }^{1,2}$; Oliveira, K.D.R. ${ }^{1,2}$; Araújo, A.L.R. ${ }^{1,2}$; Sé, A.B. ${ }^{1,2}$; Passos, R.M. ${ }^{1,2}{ }^{\text {; Hermes-Lima, M. }}{ }^{1}$

${ }^{1}$ Oxyradical Research Group, Departamento de Biologia Celular, Universidade de Brasília, Brasília, DF, 70910-900, Brazil

${ }^{2}$ Faculdade de Medicina, Universidade de Brasília

The traditional methods to teach biochemistry in most universities are based on the memorization of chemical structures, biochemical pathways and reagent names, which is many times dismotivating for the students. We presently describe an innovative, interactive and alternative method for teaching biochemistry to medical and nutrition undergraduate students, called the Biochemistry Show (BioBio Show).

The Biobio show is based on active participation of the students. They are divided in groups and the groups face each other. One group faces another one group at a time, in a game based on true or false questions that involve subjects of applied biochemistry (exercise, obesity, diabetes, cholesterol, free radicals, among others). The questions of the Show are previously elaborated by senior students. The Biobio Show has four phases, the first one is a selection exam, and from the second to the fourth phase, eliminatory confrontations happen. On a confrontation, the first group must select a certain quantity of questions for the opponent to answer. The group who choses the questions must know how to answer and justify the selected questions. This procedure is repeated on all phases of the show. On the last phase, the questions used are taken from an exam previously performed by the students: either the 9-hour biochemistry exam (Sé et al. A 9-hour biochemistry exam. An iron man competition or a good way of evaluating undergraduate students? SBBq 2005, abstract K-6) or the True-or-False exam (TFE) (Sé et al. Are tutorstudents capable of writing good biochemistry exams? SBBq 2004, abstract K-18). The winner group receives an extra 0,5 point on the final grade. Over $70 \%$ of the students informed on a questionnaire that the Biobio Show is a valuable tool for learning biochemistry. That is a new way to enrich the discussion of biochemistry in the classroom without the students getting bored. Moreover, learning is powered by critical and applied biochemistry emphasis during the Biobio Show. Financial Support: CNPq and PIBIC. 